\title{
Proceeding
}

Supplementary Issue: Summer Conferences of Sports Science. $8^{\text {th }}$ International Workshop and Conference of the International Society of Performance Analysis of Sport (ISPAS), 11-1 th of September 2019 (Budapest, Hungary) "Technology meets Practice and Science".

\section{Biomechanics of the clean and jerk in weightlifting national Jordanian team}

\author{
AMER I. AL-KHLEIFAT ${ }^{1}$, MAHER AL-KILANI ${ }^{2}$, HASHEM A. KILANI ${ }^{1}$ \\ ${ }^{1}$ School of Physical Education, University of Jordan, Amman, Jordan \\ ${ }^{2}$ Hashemite University, Zarqa, Jordan
}

\begin{abstract}
Seven elite national Jordanian weightlifters were videoed at 50 fields per second from the sagittal plane during the execution of the clean and jerk (C\&J) on an AMTI force platform with 3 weights of submaximal lifts; low medium and high weights $(35 \%, 65 \%, 85 \%)$ of their maximum. Linear and angular kinematics of the barbell with the ground reaction forces histories of the lower body were analysed using Kinova \& APAS. A significant decrease was found in the maximum vertical velocity of the barbell during the second pull of the heaviest lift $(p<0.05)$. Maximum extension velocity of the hip joint significantly increased during the first pull of the heaviest lift $(p<0.05)$. Vertical velocity and maximum bar height decreased during the second pull, especially at $85 \%$. Angle-angle diagrams of the knee and hip profile showed major differences among participants, especially at the $85 \%$ lift of their maximum. Keywords: Kinematics; Barbell; Olympic lifts.
\end{abstract}

\section{Cite this article as:}

Al-Khleifat, A.I., Al-Kilani, M., \& Kilani, H.A. (2019). Biomechanics of the clean and jerk in weightlifting national Jordanian team. Journal of Human Sport and Exercise, 14(5proc), S2429-S2434. doi:https://doi.org/10.14198/jhse.2019.14.Proc5.58

\footnotetext{
Corresponding author. School of Physical Education, University of Jordan, Amman, Jordan.

E-mail: hashem.kilani@uaeu.ac.ae

Supplementary Issue: Summer Conferences of Sports Science. $8^{\text {th }}$ International Workshop and Conference of the International Society of Performance Analysis of Sport (ISPAS), 11-13 th of September 2019 (Budapest, Hungary).

JOURNAL OF HUMAN SPORT \& EXERCISE ISSN 1988-5202

(c) Faculty of Education. University of Alicante

doi:10.14198/jhse.2019.14.Proc5.58
} 


\section{INTRODUCTION}

Analyses of barbell motion during the C\&J has been divided into a two-part lift that enables heavier loads $(\sim 18-20 \%$ greater) to be lifted than during the snatch lift (Garhammer, 1993). The clean requires the barbell to be raised from the floor (using a shoulder width grip) to the front of the shoulders in one continuous movement. The mechanical principles behind the first part of a pull include transition/double-knee bend and second pull. During the second pull of near-maximum attempts, the vertical velocity of the barbell can range from $0.88 \mathrm{~m} / \mathrm{sec}$ to $1.73 \mathrm{~m} / \mathrm{s}$ (Garhammer, 1993). In another way, the barbell lifting was dividing into stages, the force stage, the squat support stage, the stand-up stage, and the jerk stage. The Jordanian national team was recently trained and had never experience such analysis it was necessary to know the difference in the loads on the performance style between each load lifted. The maximum force values and the values of some of the biomechanical variables of the vertical and horizontal deviations from the vertical axis were analysed for the participants served in this study lifting low, medium and high weights (\%35, $\% 65$, and $\% 85$ ) of their maximum. The purpose was to compare different 3 weights loads of submaximal lifts; low medium and high weights (35\%,65\%, and $85 \%)$ of their maximum on their kinematic and kinetic disturbances.

\section{METHODS}

Seven young elite national Jordanian weightlifters at national team level (age: 21.28 years, height 175.57 $\mathrm{cm}$; weight $81.85 \mathrm{~kg}$, training age: 3.71 years) participated in the study (Table 1). All weightlifters had signed informed consent forms approved by the University Ethics Committee. Participants executed three C\&J lifts in the faculty of physical education laboratory. All trials were analysed to determine the linear kinematics of the barbell and the angular kinematics of the lower limb. Each weightlifter had 2-min between C\&J attempts for the random order of the three lifts of low, medium, and high percentages $(35 \%, 65 \%$, and $85 \%$ of their maximum). One successful C\&J trial of the three lift attempts of each subject were chosen for kinematic analysis based on the horizontal distance deviated from the vertical line of the standing posture alignment. One digital camera (Sony MiniDv PAL), captured trials at 50 fields per second was positioned perpendicular of the platform from the lifting area, forming an approximately $90^{\circ}$ angle with the sagittal plane of the weightlifters (Figure 1). The camera was 5 meters away from the lifter and 1 meter above the ground. Onemeter stick was used to calibrate the movement space. Nine linear and angular variables of the barbell kinematics of the lower body and four kinetics variables have been selected

for analyses. The little toe, ankle, knee, hip, and shoulder on the body and one point on the barbell were selected using Kinovea software at initial analyses followed by Ariel Performance Analysis System (APAS) for more accuracy.

Table 1. Characteristics of national athlete's weightlifters and barbell.

\begin{tabular}{cccccc}
\hline Subjects & Age / yrs. & Height/ $\mathbf{~ m}$ & Body mass $\mathbf{( k g})$ & Training age & Heaviest lift \\
\hline 1 & 23 & 175 & 94 & 3 & 110 \\
2 & 25 & 180 & 105 & 5 & 140 \\
3 & 16 & 175 & 69 & 3 & 90 \\
4 & 16 & 175 & 69 & 3 & 75 \\
5 & 28 & 177 & 94 & 5 & 120 \\
6 & 22 & 167 & 69 & 3 & 115 \\
7 & 19 & 180 & 73 & 4 & 130 \\
\hline
\end{tabular}




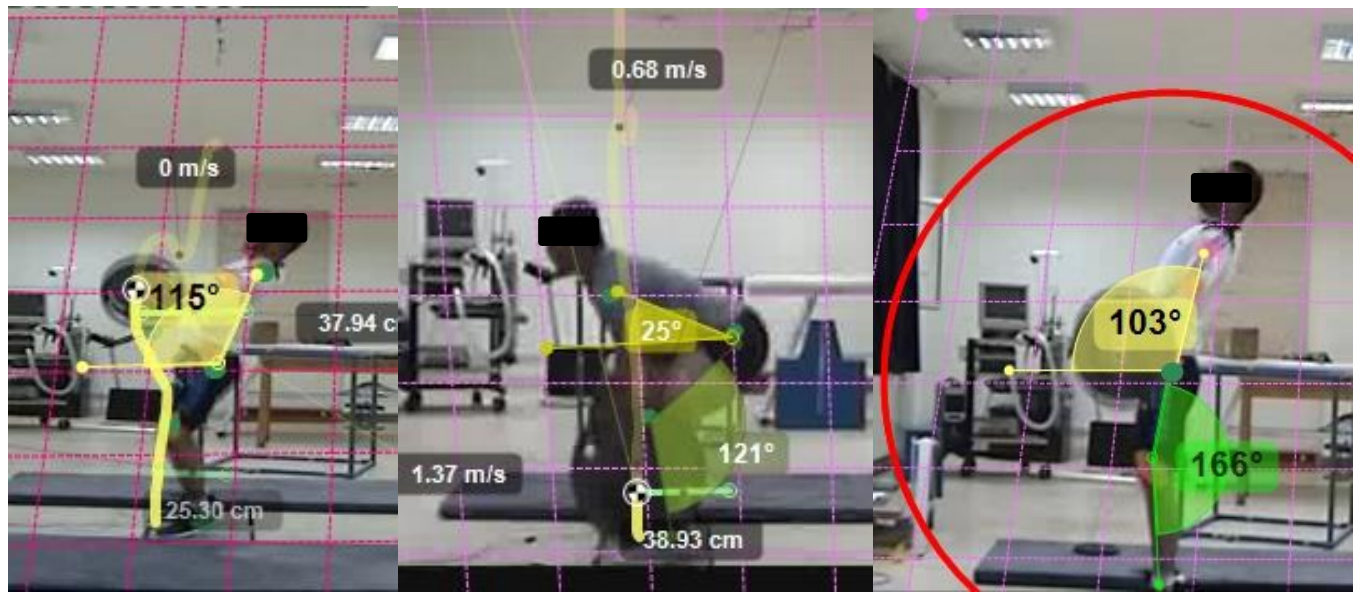

Figure 1. Experimental setup and analyses.

The following biomechanics dependent variables were considered for this analysis: Velocity of the barbell during the first pull ( $\mathrm{m} / \mathrm{s}$ ) (VFP); Horizontal distance of the barbell away from lumbar vertebrae in the first pull. (cm) (HDLFP) and second pull(HDLSP); Angle of the knee at the farthest distance of the barbell from the lumbar vertebrae in the first pull (Rad) (radian) (AKDBL); Angle of the hip during the farthest horizontal distance of the barbell from the lumbar vertebrae in the first pull (Rad) (radian) (AHDBL); Velocity of the barbell during the second pull (m/ $\mathrm{s}$ ) (VBSP); Horizontal distance from the barbell to the shoulder joint at the end of the jerk (cm) (HDBJ); Trunk angle first pull Rad (TAFP); Trunk angle second pull. Rad (TASP); Weight lifted. Kg (WL); Maximum ground reaction force (MGRF); Vertebrae moment 1st pull (VMFP); Vertebrae moment 2nd pull (VMSP) and Shoulder moment at the end of jerk (SMEJ).

The following statistical methods (SPSS) were used for means, standard deviations, Analysis of the variance with repeated measurement, post hoc, and simple linear regression analysis.

\section{RESULTS}

All selected variables are listed in table 2 for the participants in all three percentage lifts. Note that with the increase of the weight lifted, the velocity in the two pulls was less than the velocity in lifting the weight of $65 \%$ and $85 \%$ respectively, the velocity in the second pull was respectively $(2.01 \mathrm{~m} / \mathrm{s}, 1.77 \mathrm{~m} / \mathrm{s}$ and $1.56 \mathrm{~m} / \mathrm{s})$ at intensity (35\%,65\% and 85\%). By weight increase (35\%,65\% and 85\%) the mean horizontal displacement from the lumbar vertebrae in the first pull were $(39.52 \mathrm{~cm}$ and $34.19 \mathrm{~cm}$ and $30.17 \mathrm{~cm})$, and in the second pull were $(38.50 \mathrm{~cm}$ and $36.82 \mathrm{~cm}$ and $34.34 \mathrm{~cm})$ respectively.

Using repeated measures ANOVA showed that there were significant differences in all biomechanical variables which could be predicted, since the three lifts were varied in barbell mass. The results of the simple linear regression analysis for the study of the effect of the weight raised by $35 \%$ on some kinematics and kinetic variables during C\&J lift indicated a statistically significant effect of the independent variable (weight on the moment on the lumbar vertebrae within the horizontal displacement in the first pull). The value of this relationship was $(0.928)$ and the weight increased $(86.2 \%)$ of the variance in the dependent variable. The results of the simple linear regression analysis for the study of the effect of weight raised by $65 \%$ on some kinematics and kinetic variables during the C\&J lift showed a statistically significant effect of the independent variable (weight at the maximum strength) $(69.8 \%)$ of variance in the dependent variable in force and torque produced at the vertebrae and the shoulder by the end of the jerk. 
Table 2. Mean and SD of the biomechanical variables of the three barbells lifts $35 \%, 65 \%$ and $85 \%$.

\begin{tabular}{|c|c|c|c|c|c|c|}
\hline Variables & $\begin{array}{l}35 \% \\
\text { MEAN }\end{array}$ & SD & $\begin{array}{l}65 \% \\
\text { MEAN }\end{array}$ & SD & $\begin{array}{l}85 \% \\
\text { MEAN }\end{array}$ & SD \\
\hline VFP. $\mathrm{m} / \mathrm{s}^{*}$ & 1.51 & 0.23 & 1.44 & 0.04 & 1.27 & 0.09 \\
\hline HDLFP. $\mathrm{Cm}^{*}$ & 39.52 & 3.29 & 34.19 & 6.02 & 30.17 & 5.54 \\
\hline HDLSP. Cm* & 38.50 & 3.78 & 36.82 & 3.27 & 34.34 & 5.42 \\
\hline AKDBL. Rad* & 2.16 & 4.18 & 2.14 & 3.66 & 2.05 & 6.75 \\
\hline AHDBL. Rad* & 1.59 & 7.20 & 1.46 & 13.48 & 1.40 & 20.14 \\
\hline VBSP. $\mathrm{m} / \mathrm{s}^{*}$ & 2.01 & 0.08 & 1.77 & 0.07 & 1.56 & 0.06 \\
\hline HDBJ. $\mathrm{Cm}^{*}$ & 6.16 & 1.77 & 8.89 & 1.28 & 10.81 & 1.94 \\
\hline TAFP. Rad* & 0.91 & 5.06 & .84 & 6.36 & .78 & 3.28 \\
\hline TASP. Rad* & 1.55 & 7.26 & 1.70 & 9.61 & 1.82 & 12.98 \\
\hline WL. $\mathrm{Kg}^{*}$ & 39 & 8.14 & 72.57 & 20.83 & 94.42 & 34.27 \\
\hline MF. N & 1303.86 & 228.29 & 1756.00 & 301.67 & 2142.14 & 378.96 \\
\hline VMFP. $\mathrm{Nm}^{*}$ & 1340.87 & 317.09 & 2152.75 & 609.17 & 2486.24 & 780.42 \\
\hline VMSP. $\mathrm{Nm}^{*}$ & 1303.53 & 302.95 & 2323.40 & 539.70 & 2826.23 & 849.05 \\
\hline STMEJ.nm* & 207.63 & 66.64 & 560.40 & 136.82 & 884.94 & 250.95 \\
\hline
\end{tabular}

Using repeated measures ANOVA showed that there were significant differences in all biomechanical variables which could be predicted, since the three lifts were varied in barbell mass. The results of the simple linear regression analysis for the study of the effect of the weight raised by $35 \%$ on some kinematics and kinetic variables during C\&J lift indicated a statistically significant effect of the independent variable (weight on the moment on the lumbar vertebrae within the horizontal displacement in the first pull). The value of this relationship was $(0.928)$ and the weight increased $(86.2 \%)$ of the variance in the dependent variable. The results of the simple linear regression analysis for the study of the effect of weight raised by $65 \%$ on some kinematics and kinetic variables during the C\&J lift showed a statistically significant effect of the independent variable (weight at the maximum strength) (69.8\%) of variance in the dependent variable in force and torque produced at the vertebrae and the shoulder by the end of the jerk. The results of the simple linear regression analysis for the study of the effect of weight raised by $85 \%$ on some kinematics and kinetic variables during C\&J lift showed a statistically significant effect of the independent variable (weight at the maximum strength) (72.1\%) of variance in the dependent variable and the moment at the 1 st and the 2 nd pulls during this heavy lift. In Figure 1 there was a comparison in the relationships with those obtained from elite world champion (Enoka, 1988b). We saw significant variation between the lifts. We realised that this relation in the heaviest weight lifted shown in the blue dots is closer to the model.

\section{DISCUSSION}

This study is part of a large research project and follows the participants in different periods of training plan. It was difficult to discuss most of the variables and therefore was interested in what can benefit the participants in the preparatory period. Many factors can influence the outcome of a C\&J attempt. Therefore, whatever determines success is likely to be multifactorial in nature (Stone et al., 1998). The primary factors that affect the success of weightlifters in the snatch technique of weightlifting are explosive strength, flexibility, and technique (Garhammer, 1985). It was reported in previous studies that barbell trajectories could be an indicator of the effectiveness of the C\&J pull and the efficiency of the technique applied (Bruenger et al., 2007). Kinematics variables appear to be affected by high weight, causing a lack of balance on the participants' coordination and technique. It is necessary to know the best weight that can be trained to maintain the optimal barbell trajectory. We realized that the higher the weight lifted, the closer the barbell was 
from the lumbar vertebrae which were contrary to what was expected. The lower the weight lifted in the first pull in the C\&J, the farther the bar from the lumbar vertebrae, which increases the amount of movement and may lead to potential injury. High velocity in the first and second pulls at $35 \%$ of the maximum weight lifted by the participants may lead to a large motor momentum as it was supported by Gourgoulis et al., (2009), which may lead to ruptures in the muscles and ligaments (Kilani, 2015). Angle-angle diagrams profiles showed vast differences between participants at $85 \%$ lifts.

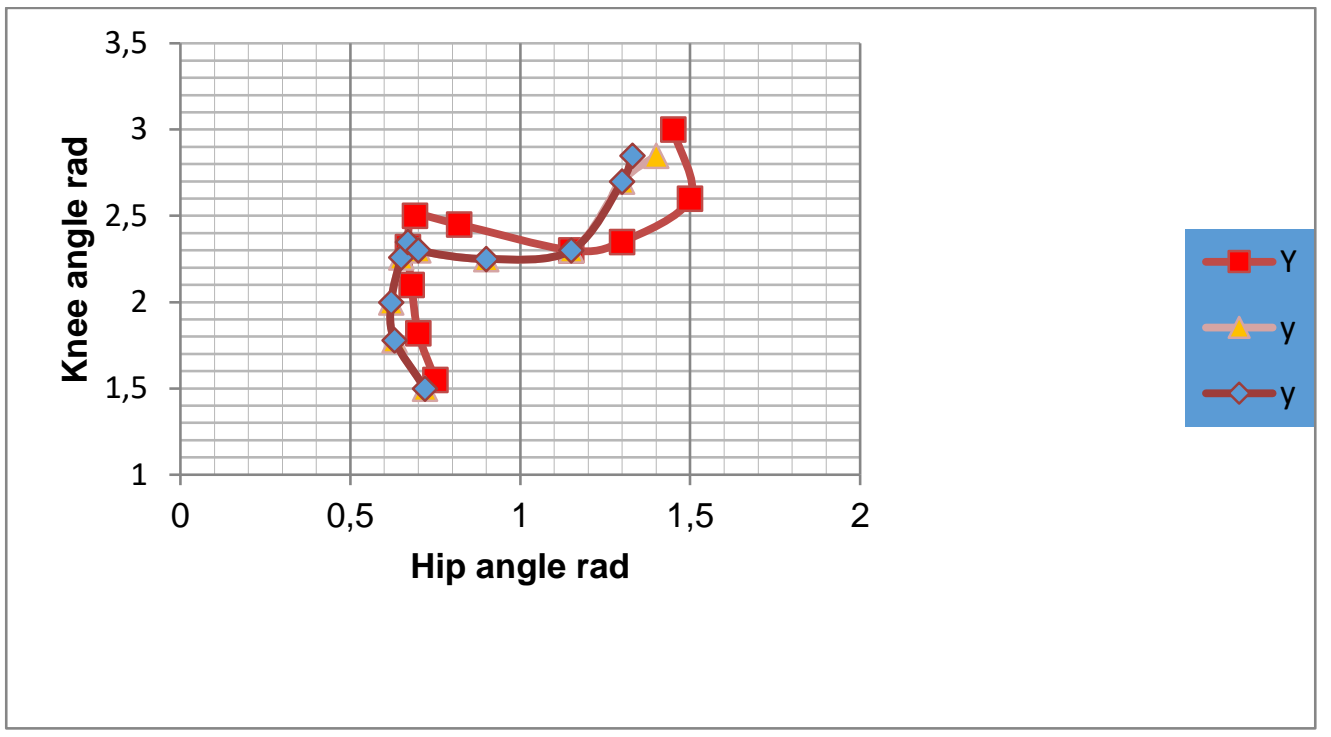

Figure 2. The variation of the relationship between knee and hip angle -angle diagram.

\section{CONCLUSION}

Coaches should start their teaching the C\&J by using light loads until they master the right technique. the closer the displacement in the first and second pulls, especially at $85 \%$ and above, provide better biomechanical efficiency to the body.

\section{REFERENCES}

Bruenger A.J., Smith S.L., Sands W.A., Leigh M.R. (2007). Validation of instrumentation to monitor dynamic performance of Olympic weightlifters. Journal of Strength and Conditioning Research 21, 492-499. https://doi.org/10.1519/00124278-200705000-00036

Garhammer, J. (1993). A review of power output studies of Olympic and Power lifting: Methodology, performance prediction, and evaluation tests. J. Strength \& Conditioning Research, 7(2): 76-89. https://doi.org/10.1519/00124278-199305000-00002

Gourgoulis V., Aggeloussis N., Garas A., Mavromatis G. (2009). Unsuccessful vs. successful performance in snatch lifts: a kinematic approach. Journal of Strength and Conditioning Research 23, 486-494. https://doi.org/10.1519/jsc.0b013e318196b843

Kilani, H. (2015) Exercise, fitness low back pain and vitamin D. Change4Life "Lack of physical activity a major risk to health. Presentation on 13 September at PDO Muscat, Oman.

Enoka RM. (1988b) Neuromechanical basis of kinesiology, Human Kinetics, Champaign. 


\section{(c) (1) (9)}

This work is licensed under a Attribution-NonCommercial-NoDerivatives 4.0 International (CC BY-NC-ND 4.0). 